Tohoku Math. J.

56 (2004), 571-582

\title{
REMARKS ON HAUSDORFF DIMENSIONS FOR TRANSIENT LIMIT SETS OF KLEINIAN GROUPS
}

\author{
Kurt FALK* AND Bernd O. Stratmann
}

(Received March 31, 2003, revised April 27, 2004)

\begin{abstract}
In this paper we study normal subgroups of Kleinian groups as well as discrepancy groups (d-groups), that are Kleinian groups for which the exponent of convergence is strictly less than the Hausdorff dimension of the limit set. We show that the limit set of a d-group always contains a range of fractal subsets, each containing the set of radial limit points and having Hausdorff dimension strictly less than the Hausdorff dimension of the whole limit set. We then consider normal subgroups $G$ of an arbitrary non-elementary Kleinian group $H$, and show that the exponent of convergence of $G$ is bounded from below by half of the exponent of convergene of $H$. Finally, we give a discussion of various examples of d-groups.
\end{abstract}

1. Introduction and statement of results. In this paper we investigate non-elementary Kleinian groups $G$ acting on $(N+1)$-hyperbolic space $\boldsymbol{D}^{N+1}$ without torsion, which have the property that their associated limit set $L(G)$ has Hausdorff dimension strictly greater than the exponent of convergence

$$
\delta(G):=\inf \left\{s \geq 0 \mid \sum_{g \in G} e^{-s \rho(0, g(0))}<\infty\right\} .
$$

(Here, $L(G)$ refers to the set of accumulation points of some $G$-orbit, and $\rho$ to the hyperbolic distance in $\boldsymbol{D}^{N+1}$ ). Throughout, we shall refer to these groups as discrepancy groups, abbreviated as d-groups.

In [6] it was shown that the limit set has positive 2-dimensional Lebesgue measure for every finitely generated, geometrically infinite d-group which acts on $\boldsymbol{D}^{3}$. This result was obtained via showing that for every arbitrary non-elementary Kleinian group $G$ one has that $\delta(G)$ coincides with the hyperbolic dimension of $G$, that is, the Hausdorff dimension of the uniformly radial limit set of $G$, or alternatively the Hausdorff dimension of the radial limit set of $G$ ([6], [26]). In this paper we consider arbitrary d-groups $G$ and discuss fractal properties of certain subsets of $L(G)$, each of which contains the radial limit set of $G$. These sets will be referred to as $\kappa$-weakly recurrent limit sets. Our first main result is that for $\kappa$ in a certain range, the Hausdorff dimension of each of these $\kappa$-weakly recurrent limit sets is strictly less than the Hausdorff dimension of $L(G)$. In particular, this also allows to specify a range of subsets of the transient limit set, the complement of the radial limit set, which have the property that their Hausdorff dimension coincides with the Hausdorff dimension of $L(G)$. Our second main result deals with the class of normal subgroups $G$ of some arbitrary non-elementary

2000 Mathematics Subject Classification. Primary 30F40; Secondary 37F35.

Key words and phrases. Kleinian groups, exponent of convergence, fractal geometry.

*Research supported by the Väisälä Foundation. 
Kleinian group $H$. For these groups it is well-known that $L(G)=L(H)$. Nevertheless, as can for instance be seen in the second example of Section 3, the hyperbolic dimension of $G$ does not necessarily coincide with the hyperbolic dimension of $H$. Here our main result is that the exponent of convergence of such a normal subgroup $G$ is always bounded from below by half of the exponent of convergence of $H$. Finally, in Section 3 we discuss various examples of d-groups. These include the infinitely-punctured Riemann surfaces of Patterson's [19, Theorem 4.4]. This type of example is closely related to the constructions of Hopf [10] and Pommerenke [22], and seems to have been the first example of a d-group in the literature. Also, we discuss the case of a normal subgroup $G$ of some convex cocompact Kleinian group $H$. If $H / G$ is non-amenable, then it follows by the work of Brooks [8] that $G$ is a d-group. Eventually, based on the further work of Patterson [20], we outline a construction of infinitely generated free d-groups of the first kind. Again, as in the normal subgroup example this construction works in any dimension, and we also show that it can be employed to construct special d-groups which have the property that the set of Jørgensen points has positive $\mathrm{N}$ dimensional spherical Lebesgue measure. These special d-groups are groups of the first kind such that the complement of their horospherical limit set contains a wandering set of positive $N$-dimensional measure. Hence, these groups do not act conservatively, and therefore they are not ergodic on $\boldsymbol{S}^{N}$ in the sense that for each of them there exists a bounded group-invariant function which is hyperbolically harmonic.

In order to state the results in detail, we now first introduce the limit sets which are relevant. Throughout, let $G$ be some arbitrary non-elementary Kleinian group without torsion. It is well-known that $L(G)$ can be decomposed into the set $L_{\mathrm{r}}(G)$ of radial limit points and the set $L_{\mathrm{t}}(G)$ of transient limit points, where

- $L_{\mathrm{r}}(G):=\left\{\xi \in L(G) \mid \liminf _{T \rightarrow \infty} \Delta\left(\xi_{T}\right)<\infty\right\}$,

- $L_{\mathrm{t}}(G):=\left\{\xi \in L(G) \mid \lim _{T \rightarrow \infty} \Delta\left(\xi_{T}\right)=\infty\right\}$.

In here, $\xi_{T}$ refers to the point on the ray from 0 to $\xi$ for which $\rho\left(0, \xi_{T}\right)=T$, and $\Delta\left(\xi_{T}\right)$ refers to the hyperbolic distance of $\xi_{T}$ to the orbit $G(0)$, that is, $\Delta\left(\xi_{T}\right):=\inf _{g \in G} \rho\left(\xi_{T}, g(0)\right)$. Important subsets of $L(G)$ are the set $L_{\mathrm{ur}}(G)$ of uniformly radial limit points and the set $L_{\mathrm{J}}(G)$ of Jørgensen limit points. These are given as follows (cf. [29], [17]).

- $L_{\mathrm{ur}}(G):=\left\{\xi \in L(G) \mid \lim \sup _{T \rightarrow \infty} \Delta\left(\xi_{T}\right)<\infty\right\}$.

- $L_{\mathrm{J}}(G)$ refers to the set of $\xi \in L(G)$ such that there exists a geodesic ray towards $\xi$ which is completely contained in some Dirichlet fundamental domain of $G$.

One easily verifies that $L_{\mathrm{ur}}(G) \subset L_{\mathrm{r}}(G)$ and that $L_{\mathrm{J}}(G) \subset L_{\mathrm{t}}(G)$. Note that for ease of exposition we have defined the set $L_{\mathrm{J}}(G)$ so that the set of bounded parabolic fixed points of $G$ is contained in $L_{\mathrm{J}}(G)$ (for the definition of a bounded parabolic fixed point we refer to [16, p. 43]), and hence our definition of $L_{\mathrm{J}}(G)$ here differs from the definition given in [17]. Also, note that $L_{\mathrm{J}}(G)$ corresponds to the dissipative part of the action of $G$ on the sphere at infinity (cf. [30], [14]).

Finally, we introduce the set $L_{\mathrm{t}}^{(\kappa)}(G)$ of $\kappa$-transient limit points and the set $L_{\mathrm{r}}^{(\kappa)}(G)$ of $\kappa$-weakly recurrent limit points for $\kappa>0$ as follows. 
- $L_{\mathrm{r}}^{(\kappa)}(G):=\bigcup_{c>0}\left\{\begin{array}{l|l}\xi \in L(G) & \begin{array}{l}\xi \in b\left(\Pi(g(0)), c e^{-\rho(0, g(0)) /(1+\kappa)}\right) \\ \text { for infinitely many } g \in G\end{array}\end{array}\right\}$.

- $L_{\mathrm{t}}^{(\kappa)}(G):=L(G) \backslash L_{\mathrm{r}}^{(\kappa)}(G)$.

In here, $b(\eta, r) \subset S^{N}$ refers to the ball centred at $\eta \in S^{N}$ of spherical radius $r$, and $\Pi$ to the shadow projection from zero to the boundary $S^{N}$ of the hyperbolic space. One easily verifies that $L_{\mathrm{t}}^{\left(\kappa_{1}\right)}(G) \supset L_{\mathrm{t}}^{\left(\kappa_{2}\right)}(G)$ whenever $\kappa_{1} \leq \kappa_{2}$, and that $L_{\mathrm{r}}(G) \subset L_{\mathrm{r}}^{(\kappa)}(G)$ for all $\kappa>0$. Also, note that $L_{\mathrm{r}}(G)$ is a dense subset of $L(G)$, and hence so is $L_{\mathrm{r}}^{(\kappa)}(G)$. Therefore, by a standard result in fractal geometry (see e.g. [9]), it follows that the lower packing dimension of $L_{\mathrm{r}}^{(\kappa)}(G)$ coincides with the lower packing dimension of $L(G)$, where the latter is always greater than or equal to the Hausdorff dimension of $L(G)$. The following theorem shows that the Hausdorff dimension of $L_{\mathrm{r}}^{(\kappa)}(G)$ relates in a more subtle way to the Hausdorff dimension $\operatorname{dim}_{H}(L(G))$ of $L(G)$. The theorem gives the first main result of the paper.

THEOREM 1. Let $G$ be a d-group. With $\delta_{*}(G):=\left(\operatorname{dim}_{H}(L(G))-\delta(G)\right) / \delta(G)$, we have for all $0<\kappa<\delta_{*}(G)$,

$$
\delta(G) \leq \operatorname{dim}_{\mathrm{H}}\left(L_{\mathrm{r}}^{(\kappa)}(G)\right)<\operatorname{dim}_{\mathrm{H}}(L(G))
$$

and in particular

$$
\operatorname{dim}_{\mathrm{H}}\left(L_{\mathrm{t}}^{(\kappa)}(G)\right)=\operatorname{dim}_{\mathrm{H}}(L(G)) .
$$

Note that by a result of Beardon ([2], [3]) the exponent of convergence of a non-elementary Kleinian group is strictly positive, which gives that $\delta_{*}(G)$ is well-defined.

Our second main result considers normal subgroups of an arbitrary Kleinian group. We refer to Section 3 (Example 2) for a discussion of some examples for d-groups of this type.

THEOREM 2. Let $H$ be a non-elementary Kleinian group, and let $G$ be a non-trivial normal subgroup of $H$. We then have

$$
\delta(G) \geq \frac{\delta(H)}{2} .
$$

Before giving the proofs of these theorems, let us first show that they have some interesting immediate implications. For the first recall that a Kleinian group $G$ is said to be of $\delta(G)$ convergence type if $\sum_{g \in G} e^{-\delta(G) \rho(0, g(0))}$ converges. Also, let $\mathcal{H}^{s}$ refer to the $s$-dimensional Hausdorff measure. Note that the statement (i) in the following corollary generalizes a result in [5, Corollary 5], where the case $\operatorname{dim}_{\mathrm{H}}(L(G))=N$ has been considered.

COROLlARY 1. For each d-group $G$ the following holds.

(i) If $\mathcal{H}^{\operatorname{dim}_{\mathrm{H}}(L(G))}(L(G))>0$, then $\mathcal{H}^{\operatorname{dim}_{\mathrm{H}}(L(G))}\left(L_{\mathrm{t}}^{(\kappa)}(G)\right)=\mathcal{H}^{\operatorname{dim}_{\mathrm{H}}(L(G))}(L(G))$ for all $0<\kappa<\delta_{*}(G)$.

(ii) If $G$ is of $\delta(G)$-convergence type, then $\mathcal{H}^{(1+\kappa) \delta(G)}\left(L_{\mathrm{r}}^{(\kappa)}(G)\right)=0$ for all $0<\kappa \leq$ $\delta_{*}(G)$. 
The following corollary represents the main theorem of [5]. We remark that the work in this paper was originally inspired by this result of Bishop. We also refer to Lundh's paper [11].

COROLLARY 2. For every non-elementary Kleinian group $G$ we have

$$
\operatorname{dim}_{\mathrm{H}}(L(G))=\max \left(\delta(G), \operatorname{dim}_{\mathrm{H}}\left(\bigcup_{\kappa>0} L_{\mathrm{t}}^{(\kappa)}(G)\right)\right) .
$$

Finally, let us briefly comment on the way our results relate to horospherical limit sets (recall that $\xi \in L(G)$ is called a horospherical limit point if every horoball at $\xi$ contains infinitely many elements of $G(0))$. In [31] Tukia introduced the so-called big horospherical limit set, which consists of limit points $\xi \in L(G)$ for which there exists a horoball at $\xi$ containing infinitely many elements of $G(0)$. One can verify that every horospherical limit point is contained in the big horospherical limit set, and that $L_{\mathrm{r}}^{(1)}(G)$ coincides with the big horospherical limit set (see the proof of Lemma 2). A straightforward adaptation of the proof of Theorem 1 (where one has to replace $L(G)$ by $L_{\mathrm{r}}^{(1)}(G)$ ) then gives rise to the following proposition.

Proposition. Let $G$ be a non-elementary Kleinian group such that $\delta(G)<$ $\operatorname{dim}_{\mathrm{H}}\left(L_{\mathrm{r}}^{(1)}(G)\right)$. We then have for all $0<\tau<\left(\operatorname{dim}_{\mathrm{H}}\left(L_{\mathrm{r}}^{(1)}(G)\right)-\delta(G)\right) / \delta(G)$,

$$
\operatorname{dim}_{\mathrm{H}}\left(L_{\mathrm{r}}^{(\tau)}(G)\right)<\operatorname{dim}_{\mathrm{H}}\left(L_{\mathrm{r}}^{(1)}(G)\right) .
$$

We would like to thank the Department of Mathematics at the University of Helsinki for warm hospitality and financial support. Also, we are grateful to Pekka Tukia for helpful conversations on the construction of certain d-groups, as well as to the referee for his/her careful reading of the original manuscript and for the helpful comments which significantly improved the paper. Finally, the second author would like to thank the Mathematical Institute and the Institute for Mathematical Stochastics at the University of Göttingen for hospitality and excellent working conditions.

\section{Proofs.}

2.1. Upper bounds for the Hausdorff dimension of weakly recurrent limit sets.

Proof of Theorem 1. Let $\sigma>0$ be given. By definition, $L_{\mathrm{r}}^{(\sigma)}(G)$ can be written as a union of limsup-sets as follows

$$
L_{\mathrm{r}}^{(\sigma)}(G)=\bigcup_{c>0} \lim \sup \left\{b\left(\Pi(g(0)), c e^{-\rho(0, g(0)) /(1+\sigma)}\right) \mid g \in G\right\} .
$$

For each $c>0$ the family $\left\{b\left(\Pi(g(0)), c e^{-\rho(0, g(0)) /(1+\sigma)}\right) \mid g \in G\right\}$ represents a covering of $\lim \sup \left\{b\left(\Pi(g(0)), c e^{-\rho(0, g(0)) /(1+\sigma)}\right) \mid g \in G\right\}$. For the radii of these covering balls we have by the definition of $\delta(G)$ that

$$
\sum_{g \in G}\left(c e^{-\rho(0, g(0)) /(1+\sigma)}\right)^{s}<\infty \text { for all } s>(1+\sigma) \delta(G) .
$$


Therefore, the $s$-dimensional Hausdorff measure of the limsup-set associated with $c$ is finite for all $s>(1+\sigma) \delta(G)$, which gives

$$
\operatorname{dim}_{\mathrm{H}}\left(\lim \sup \left\{b\left(\Pi(g(0)), c e^{-\rho(0, g(0)) /(1+\sigma)}\right) \mid g \in G\right\}\right) \leq(1+\sigma) \delta(G) .
$$

Since $c$ was chosen to be arbitrary, the latter estimate clearly holds for every $c>0$. By the monotonicity of Hausdorff dimension (see e.g. [9, p.29]), we therefore have

$$
\operatorname{dim}_{\mathrm{H}}\left(L_{\mathrm{r}}^{(\sigma)}(G)\right) \leq(1+\sigma) \delta(G) .
$$

This immediately implies that for every $\sigma$ with $(1+\sigma) \delta(G)<\operatorname{dim}_{H}(L(G))$, or what is equivalent for every $\sigma<\delta_{*}$, we have

$$
\operatorname{dim}_{\mathrm{H}}\left(L_{\mathrm{r}}^{(\sigma)}(G)\right)<\operatorname{dim}_{\mathrm{H}}(L(G)) .
$$

This proves the first assertion of the theorem. The second assertion is an immediate consequence of the first. Namely, as we have just shown $\operatorname{dim}_{\mathrm{H}}\left(L_{\mathrm{r}}^{(\kappa)}(G)\right)<\operatorname{dim}_{\mathrm{H}}(L(G))$ for all $0<\kappa<\delta_{*}$, and hence for $\kappa$ in this range we have

$$
\operatorname{dim}_{\mathrm{H}}(L(G))=\operatorname{dim}_{\mathrm{H}}\left(L(G) \backslash L_{\mathrm{r}}^{(\kappa)}(G)\right)=\operatorname{dim}_{\mathrm{H}}\left(L_{\mathrm{t}}^{(\kappa)}(G)\right) .
$$

Proofs of Corollaries. Corollary 2 is an immediate consequence of Theorem 1. For Corollary 1 (i), Theorem 1 gives $\operatorname{dim}_{H}\left(L_{\mathrm{r}}^{(\kappa)}(G)\right)<\operatorname{dim}_{H}(L(G))$, for all $0<\kappa<$ $\delta_{*}(G)$. For $\kappa$ in this range we thus have that if $\mathcal{H}^{\operatorname{dim}_{H}(L(G))}(L(G))>0$, then $\mathcal{H}^{\operatorname{dim}_{H}(L(G))}(L(G))=$ $\mathcal{H}^{\operatorname{dim}_{\mathrm{H}}^{(L(G))}}\left(L_{\mathrm{t}}^{(\kappa)}(G)\right)>0$.

Corollary 1 (ii) is proved by way of contradiction. Assume that $\mathcal{H}^{(1+\kappa) \delta(G)}\left(L_{\mathrm{r}}^{(\kappa)}(G)\right)>0$ for $0<\kappa \leq \delta_{*}(G)$. Using Frostman's lemma (cf. [15]), it follows that there exists a finite Radon measure $v_{\kappa}$ with compact support in $L_{\mathrm{r}}^{(\kappa)}(G)$, such that $v_{\kappa}(b(\eta, R)) \leq R^{(1+\kappa) \delta(G)}$ for all $\eta \in S^{N}, R>0$. Using the definition of $\delta(G)$ and the fact that $G$ is of $\delta(G)$-convergence type, it follows for all $c>0$,

$$
\sum_{g \in G} v_{\kappa}\left(b\left(\Pi(g(0)), c e^{-\rho(0, g(0)) /(1+\kappa)}\right)\right)<\infty .
$$

By the Borel-Cantelli lemma, we hence have for all $c>0$,

$$
\nu_{\kappa}\left(\lim \sup \left\{b\left(\Pi(g(0)), c e^{-\rho(0, g(0)) /(1+\kappa)}\right)\right\}\right)=0 .
$$

This implies $v_{\kappa}\left(L_{\mathrm{r}}^{(\kappa)}(G)\right)=0$, and hence gives a contradiction.

2.2. A lower bound for the exponent of convergence of normal subgroups.

In order to prepare for the proof of Theorem 2 we give the following elementary geometric estimates. In here $B(z, r)$ refers to the open hyperbolic ball centred at $z \in \boldsymbol{D}^{N+1}$ of radius $r$, and $|\Pi(E)|:=\mid\left\{\xi \in S^{N} \mid \xi_{T} \in E\right.$ for some $\left.T>0\right\} \mid$ denotes the spherical diameter of the shadow projection $\Pi(E)$ of $E \subset D^{N+1}$ from zero to the boundary $\boldsymbol{S}^{N}$ of the hyperbolic space. Also, we use the common convention $a \asymp b$ to describe that the ratio of two positive real numbers $a$ and $b$ is uniformly bounded away from zero and infinity. 


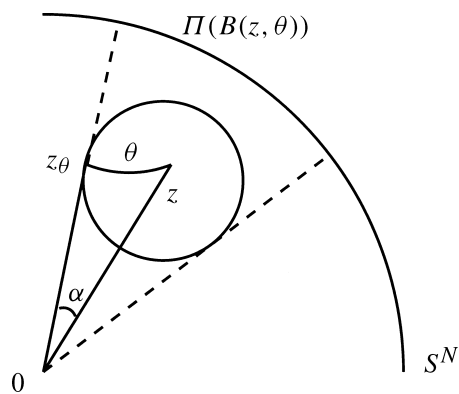

FIGURE 1. The setting of Lemma 1.

LEMMA 1. Let $\kappa>0$ be given. For all $z \in D^{N+1}$ such that $\rho(0, z)$ is sufficiently large, we have

$$
\left|\Pi\left(B\left(z, \frac{\kappa}{1+\kappa} \rho(0, z)\right)\right)\right| \asymp e^{-\rho(0, z) /(1+\kappa)} .
$$

Proof. Define $\theta:=\kappa \rho(0, z) /(1+\kappa)$, and let $z_{\theta}$ refer to the point of tangency of some geodesic ray which starts at the origin and which is tangential to the boundary of $B(z, \theta)$. Consider the right-angled triangle with vertices $0, z$ and $z_{\theta}$, and let $\alpha$ denote its angle at 0 (see Figure 1). Using the 'hyperbolic cosine rule' [4, p. 148] we have

$$
e^{\rho\left(0, z_{\theta}\right)} \asymp e^{\rho(0, z)} e^{-\theta}=e^{\rho(0, z)} e^{-\kappa \rho(0, z) /(1+\kappa)}=e^{\rho(0, z) /(1+\kappa)} .
$$

Also, by the 'hyperbolic tangent rule' for right-angled triangles [4, p. 147] we have

$$
\tanh \theta=\sinh \rho\left(0, z_{\theta}\right) \tan \alpha .
$$

Furthermore, note that for $\rho(0, z)$ sufficiently large so that $\alpha$ is bounded away from $\pi / 2$, we have

$$
|\Pi(B(z, \theta))| \asymp \tan \alpha .
$$

Combining these three observations, we deduce

$$
|\Pi(B(z, \kappa \rho(0, z) /(1+\kappa)))| \asymp \tan \alpha=\frac{\tanh \theta}{\sinh \rho\left(0, z_{\theta}\right)} \asymp e^{-\rho\left(0, z_{\theta}\right)} \asymp e^{-\rho(0, z) /(1+\kappa)} .
$$

For the proof of Theorem 2 we also require the following result of Matsuzaki [13, Theorem 6], for which we include a proof. Lemma 2 shows in particular that the radial limit set of any arbitrary Kleinian group $H$ is always a subset of the big horospherical limit set of any normal subgroup of $H$, since it is implicit from the proof that the big horospherical limit set of any Kleinian group $G$ coincides with $L_{\mathrm{r}}^{(1)}(G)$. 
LEMMA 2. Let $H$ be a non-elementary Kleinian group, and let $G$ be a non-trivial normal subgroup of $H$. We then have

$$
L_{\mathrm{r}}(H) \subset L_{\mathrm{r}}^{(1)}(G) \subset L(H) .
$$

Proof. We clearly have that $L_{\mathrm{r}}^{(1)}(G)$ is a subset of $L(G)$. Since $L(G)=L(H)$, it is therefore sufficient to show that $L_{\mathrm{r}}(H) \subset L_{\mathrm{r}}^{(1)}(G)$. For this, let $\xi$ be some arbitrary element of $L_{\mathrm{r}}(H)$. Then there exists a sequence $\left(h_{n}\right)$ of elements $h_{n} \in H$ such that $h_{n}(0)$ approaches $\xi$ conically, that is, $h_{n}(0)$ tends to $\xi$ and there exists $c>0$ such that $\xi \in \Pi\left(B\left(h_{n}(0), c\right)\right)$ for all $n \in N$. With $g_{0} \in G \backslash\{$ id. $\}$ referring to some fixed element, we have that $h_{n} g_{0} h_{n}^{-1} \in G$, for all $n \in N$. Using the triangle inequality, we obtain

$$
\rho\left(h_{n}(0), h_{n} g_{0} h_{n}^{-1}(0)\right)=\rho\left(0, g_{0} h_{n}^{-1}(0)\right) \leq \rho\left(0, g_{0}(0)\right)+\rho\left(0, h_{n}(0)\right) .
$$

Hence, with $H_{\xi}$ referring to the horoball at $\xi$ such that $0 \in H_{\xi}$ and that 0 has hyperbolic distance $c_{0}:=\rho\left(0, g_{0}(0)\right)+2 c$ to the horospherical boundary of $H_{\xi}$, the latter estimate implies that $\left\{h_{n} g_{0} h_{n}^{-1}(0) \mid n \in N\right\} \subset H_{\xi}$. Now observe that, by Lemma 1 and by a wellknown estimate concerning hyperbolic geometry within horoballs (see e.g. [25, Lemma 2]), we have that a hyperbolic ball, which is tangential to the ray from the origin to $\xi$ and which is centred at some arbitrary $z \in H_{\xi}$, must have hyperbolic radius not exceeding $c_{0}+\rho(0, z) / 2$. Therefore,

$$
\xi \in \Pi\left(B\left(h_{n} g_{0} h_{n}^{-1}(0), \frac{\rho\left(0, h_{n} g_{0} h_{n}^{-1}(0)\right)}{2}+c_{0}\right)\right) \text { for all } n \in N .
$$

Using Lemma 1, it then follows that $\xi \in L_{\mathrm{r}}^{(1)}(G)$.

Proof of Theorem 2. For $G$ such that $\delta(G)=\operatorname{dim}_{H}(L(G))$ the statement of the theorem is trivial. Hence, we can assume without loss of generality that $G$ is a d-group. Assume by way of contradiction that there exists $\tau>0$ such that $2 \delta(G)+\tau<\delta(H)$. Let $\varepsilon>0$ be sufficiently small so that $\tau-2 \varepsilon>0$, and then choose $\sigma$ so that $0<\sigma<\tau-2 \varepsilon$. With these choices we have that $\delta(G)+\varepsilon<(\delta(H)-\sigma) / 2$, and therefore

$$
\sum_{g \in G}\left(e^{-\rho(0, g(0)) / 2}\right)^{\delta(H)-\sigma} \leq \sum_{g \in G}\left(e^{-\rho(0, g(0))}\right)^{\delta(G)+\varepsilon}<\infty .
$$

Hence, we have for all $c>0$,

$$
\operatorname{dim}_{\mathrm{H}}\left(\lim \sup \left\{b\left(\Pi(g(0)), c e^{-\rho(0, g(0)) / 2}\right) \mid g \in G\right\}\right) \leq \delta(H)-\sigma,
$$

which gives $\operatorname{dim}_{\mathrm{H}}\left(L_{\mathrm{r}}^{(1)}\right) \leq \delta(H)-\sigma$. Using Lemma 2, it now follows

$$
\delta(H)=\operatorname{dim}_{\mathrm{H}}\left(L_{\mathrm{r}}(H)\right) \leq \operatorname{dim}_{\mathrm{H}}\left(L_{\mathrm{r}}^{(1)}(G)\right) \leq \delta(H)-\sigma,
$$

which gives a contradiction.

3. Some examples. In this section we discuss some examples of d-groups. For further interesting examples of d-groups we refer to [12]. 
EXAMPLE 1. ('Infinitely-punctured Riemann surfaces') The first example represents a simply connected Riemann surface with infinitely many punctures. The example is due to Patterson [19, Theorem 4.4], and to our knowledge it has been the first example of a d-group in the literature. Here, we only give a brief description of the construction of this type of Fuchsian groups, and we refer to [19] for the proof that these groups are in fact d-groups (the proof in [19] uses uniformization theory in combination with perturbation theory of the Laplacian).

Let $G_{0}$ be a cocompact Fuchsian group acting on $\boldsymbol{D}^{2}$ without elliptic elements. Then $\left(\boldsymbol{D}^{2} \backslash G_{0}(0)\right) / G_{0}$ is a compact Riemann surface with one puncture, and hence it is conformally isomorphic to $\boldsymbol{D}^{2} / G_{1}$, for some cofinite Fuchsian $G_{1}$ with exactly one parabolic element. Consider the canonical group homomorphism $\phi: G_{1} \rightarrow G_{0}$, and let $G:=\operatorname{ker}(\phi)$. Clearly, $G$ is a normal subgroup of $G_{1}$ and uniformizes $\boldsymbol{D}^{2} \backslash G_{0}(0)$. In [19] it was shown that $G$ is a group of the first kind for which $\delta(G)<1$. Hence, it follows that $G$ is a d-group.

EXAMPLE 2. ('Normal subgroups') The second example is mainly based on an application of a beautiful result of Brooks in [8], who gave a significant extension of results of Rees [23], [24] (see also [32] and the discussion in [21]).

Let $G_{0}$ and $G_{1}$ be two non-elementary convex cocompact Kleinian groups acting on $\boldsymbol{D}^{N+1}$ with (open) fundamental domains $F_{0}$ and $F_{1}$, respectively, such that $\bar{F}_{0} \cap \bar{F}_{1}=\emptyset$. For simplicity, we assume that $G_{0}$ is freely generated by hyperbolic automorphisms $g_{1}, \ldots, g_{k}$, and likewise that $G_{1}$ is freely generated by hyperbolic automorphisms $g_{k+1}, \ldots, g_{k+n}$ (for $k, n>1)$. With $H:=G_{0} * G_{1}$ referring to the free product of $G_{0}$ and $G_{1}$, we also assume that $\delta(H)>N / 2$. Let $\varphi: H \rightarrow G_{1}$ denote the canonical group homomorphism, and define $G:=\operatorname{ker}(\varphi)$. It is easily verified that $G=\left\langle h g_{i} h^{-1} \mid i=1, \ldots, k, h \in G_{1}\right\rangle$, and that $G$ is the normal subgroup of $H$ generated by $G_{0}$ in $H$. Hence, it follows that $H / G$ is isomorphic to $G_{1}$. In order to see that $G$ is a d-group, recall that Brooks [8] has shown that if $\Gamma_{2}$ is a nontrivial normal subgroup of a convex cocompact Kleinian group $\Gamma_{1}$ with $\delta\left(\Gamma_{1}\right)>N / 2$, then we have that $\delta\left(\Gamma_{1}\right)=\delta\left(\Gamma_{2}\right)$ if and only if $\Gamma_{1} / \Gamma_{2}$ is amenable (for the notion 'amenable' see e.g. [7], [34]). Observe that in our example here we have that $H / G$ contains a free subgroup on two generators, and therefore $H / G$ is not amenable (note that every group which contains a free group with two generators is necessarily non-amenable (see e.g. [33])). Hence, applying the result of Brooks, it follows that $G$ is a d-group.

EXAMPLE 3. ('Cantor-tree endings made of cylinders') The third example gives an infinitely generated d-group of the first kind which acts on $\boldsymbol{D}^{N+1}$. In particular, these groups give rise for instance to geometrically infinite hyperbolic $(N+1)$-manifolds without cusps, which consist of a 'cocompact root' and an attached ending which is basically an 'infinite capstan of hyperbolic cylinders' (see Figure 2). Our construction gives a slight modification of the construction of Patterson in [20] (see also [1]). We have simplified the original construction in [20] (paragraph 5) in order to make the ideas more transparent. 


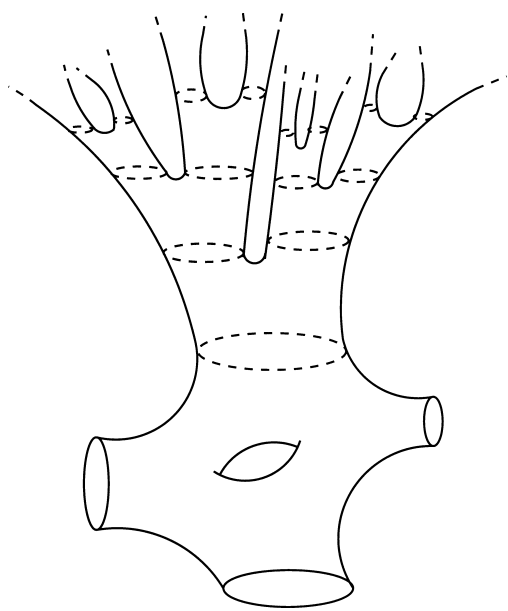

FIGURE 2. Cantor-tree endings made of hyperbolic cylinders.

Let us first recall from [20] the following observation relating the exponent of convergence of a convex cocompact Kleinian group $\Gamma$ to the exponent of convergence of the free product $\Gamma *\langle\gamma\rangle$, for some suitably chosen hyperbolic transformation $\gamma$.

For $\xi \in S^{N}$, let $\mathcal{H}_{\xi}$ denote the set of all hyperbolic automorphisms of $\boldsymbol{D}^{N+1}$ which have $\xi$ as a fixed point. For $\gamma \in \mathcal{H}_{\xi}$, let $F_{\gamma}$ refer to the Dirichlet fundamental domain for $\langle\gamma\rangle$ (constructed with respect to $0 \in F_{\gamma}$ ). We then have that $F_{\gamma}$ is bounded by two disjoint hyperplanes $H_{1}(\gamma)$ and $H_{2}(\gamma)$ of codimension one, and we let $\mathcal{H}_{\xi}^{*}$ denote the set of those elements of $\mathcal{H}_{\xi}$ for which these two hyperplanes are of equal Euclidean size.

Let $F$ be the Dirichlet fundamental domain for the convex cocompact group $\Gamma$ (constructed with respect to $0 \in F$ ). Then fix some arbitrary point $\omega$ contained in some connected component $\Omega$ of $\bar{F} \cap S^{N}$, and let $\mathcal{H}_{\omega}^{*}(\Omega)$ refer to the set of elements $\gamma \in \mathcal{H}_{\omega}^{*}$ for which $\Pi\left(H_{1}(\gamma) \cup H_{2}(\gamma)\right) \subset \Omega$. With these preparations we then have (cf. [20])

$$
\delta(\Gamma *\langle\gamma\rangle) \rightarrow \delta(\Gamma) \quad \text { for } \quad \gamma \in \mathcal{H}_{\omega}^{*}(\Omega) \text { such that }\left|\Pi\left(H_{1}(\gamma)\right)\right| \rightarrow 0 .
$$

The idea of the proof of this statement is roughly as follows (we refer to [20] for the details). Recall that the limit set $L(\Gamma)$ is constructed very much like a Cantor set generated by a certain set of contractions. Likewise, $L(\Gamma *\langle\gamma\rangle)$ is generated by the same set of contractions together with some additional contractions, which correspond to $\gamma$ and $\gamma^{-1}$. It is intuitively clear that for $\left|\Pi\left(H_{1}(\gamma)\right)\right| \rightarrow 0$, the amount of contraction of these additional generators becomes arbitrarily large, and therefore, in the limit the Hausdorff dimension cannot increase.

With this preliminary observation we can now construct the following class of d-groups. Let $G_{0}$ be some fixed convex cocompact Kleinian group acting on $\boldsymbol{D}^{N+1}$ such that $\tau_{0}:=$ $\delta\left(G_{0}\right)<N$. Fix some number $\tau_{0}<\tau<N$, as well as some strictly increasing sequence $\left(\tau_{k}\right)_{k=0,1,2, \ldots}$ of numbers $\tau_{k}$ such that $\lim \tau_{k}=\tau$. With $F_{0}$ referring to a Dirichlet fundamental 
domain of $G_{0}$ (constructed with respect to $0 \in F_{0}$ ), we let $\mathcal{O}_{0}$ denote the set of connected components of $\bar{F}_{0} \cap \boldsymbol{S}^{N}$. Also, fix some countable set $X=\left\{\xi_{1}, \xi_{2}, \ldots\right\}$ which is dense in $\bigcup_{\Omega \in \mathcal{O}_{0}} \Omega$. That is, we let $X \subset \bigcup_{\Omega \in \mathcal{O}_{0}} \Omega$ and $\bar{X}=\bigcup_{\Omega \in \mathcal{O}_{0}} \bar{\Omega}$.

We can then construct a sequence $\left(G_{k}\right)_{k=0,1, \ldots}$ of convex cocompact groups $G_{k}$ by way of induction as follows. In here, $F_{k}$ refers to the Dirichlet fundamental domain of $G_{k}$ (constructed with respect to $0 \in F_{k}$ ), and $\mathcal{O}_{k}$ denotes the set of connected components of $\bar{F}_{k} \cap \boldsymbol{S}^{N}$. Now, if $G_{k-1}$ is given for some $k \in N$, then $G_{k}$ is obtained as follows.

If $\xi_{k} \in L\left(G_{k-1}\right)$, then we let $G_{k}=G_{k-1}$. Otherwise, i.e. for $\xi_{k} \notin L\left(G_{k-1}\right)$, there exist $g_{k} \in G_{k-1}$ and $\Omega \in \mathcal{O}_{k-1}$ such that $g_{k}\left(\xi_{k}\right) \in \Omega$. Hence, by the observation above, there exists $\gamma_{k} \in \mathcal{H}_{g_{k}\left(\xi_{k}\right)}^{*}(\Omega)$ such that $\delta\left(G_{k-1} *\left\langle\gamma_{k}\right\rangle\right) \leq \tau_{k}$. In this situation, we then let

$$
G_{k}=G_{k-1} *\left\langle\gamma_{k}\right\rangle .
$$

In this way we obtain the sequence $\left(G_{k}\right)$ of convex cocompact groups, and we define

$$
G:=\bigcup_{k=0}^{\infty} G_{k}
$$

In order to see that $G$ is a d-group, recall that Sullivan ([28]; see also Remark 1 below) has shown that if $\Gamma_{1} \subset \Gamma_{2} \subset \cdots \subset \Gamma_{k} \subset \cdots$ is an increasing sequence of subgroups of the Kleinian group $\Gamma=\bigcup_{k} \Gamma_{k}$, then it follows that $\delta(\Gamma)=\sup _{k} \delta\left(\Gamma_{k}\right)$. Applying this result to our sequence $\left(G_{k}\right)$ here, we obtain

$$
\delta(G)=\delta\left(\bigcup G_{k}\right)=\sup \delta\left(G_{k}\right) \leq \sup \tau_{k}=\tau .
$$

Also note that by construction we have that $\left\{\xi_{1}, \ldots, \xi_{k}\right\} \subset L\left(G_{k}\right) \cap \bigcup_{\Omega \in \mathcal{O}_{0}} \Omega$, for each $k \in N$. This implies that $X \subset L(G) \cap \bigcup_{\Omega \in \mathcal{O}_{0}} \Omega$, and hence, since $X$ is dense in $\bigcup_{\Omega \in \mathcal{O}_{0}} \Omega$ (and thus $G_{0}(X)$ is dense in $S^{N}$ ), it follows that $L(G)$ is dense in $S^{N}$. Using the fact that $L(G)$ is closed, it then follows that $L(G)=S^{N}$, and hence that $G$ is a Kleinian group of the first kind. Summarizing the above, we now have that

$$
\delta(G) \leq \tau<N=\operatorname{dim}_{H}(L(G)),
$$

which gives that $G$ is a d-group.

REMARK 1 (see also [27]). The proof in [28] of Sullivan's result which we employed in Example 3 mainly uses the conformality of the Patterson measure. It seems worth mentioning that this result can be derived alternatively by purely elementary means as follows. One easily verifies that $\bigcup_{k} L_{\text {ur }}\left(\Gamma_{k}\right) \subset L_{\text {ur }}(\Gamma)$. On the other hand, if $\xi \in L_{\text {ur }}(\Gamma)$, then there exists an infinite path $p_{\xi}$ in the Cayley graph of $\Gamma$ such that the ray from the origin to $\xi$ is fully contained in some fixed hyperbolic neighbourhood of $p_{\xi}$, and that the hyperbolic lengths of the geodesic segments of $p_{\xi}$ are uniformly bounded from above. Therefore $L_{\mathrm{ur}}(\Gamma)=\bigcup_{k} L_{\mathrm{ur}}\left(\Gamma_{k}\right)$. Using the monotonicity of Hausdorff dimension (see e.g. [9]) and the fact that $\delta(H)=\operatorname{dim}_{\mathrm{H}}\left(L_{\mathrm{ur}}(H)\right)$ for every non-elementary Kleinian group $H$ ([6], [26]), it 
follows that

$$
\begin{aligned}
\delta(\Gamma) & =\operatorname{dim}_{\mathrm{H}}\left(L_{\mathrm{ur}}(\Gamma)\right)=\operatorname{dim}_{\mathrm{H}}\left(\bigcup_{k} L_{\mathrm{ur}}\left(\Gamma_{k}\right)\right) \\
& =\sup _{k} \operatorname{dim}_{\mathrm{H}}\left(L_{\mathrm{ur}}\left(\Gamma_{k}\right)\right)=\sup _{k} \delta\left(\Gamma_{k}\right) .
\end{aligned}
$$

REMARK 2. It is straightforward to refine the latter construction to obtain a dgroup $G$ which has the property that the $N$-dimensional spherical Lebesgue measure $\lambda_{N}\left(L_{\mathrm{J}}(G)\right)$ of the set of Jørgensen points is strictly positive. In order to obtain such a group, one proceeds as follows. Let $\left(\theta_{k}\right)_{k \in N}$ denote some sequence of positive numbers such that $\sum_{k \in N} \theta_{k}<1 / 2$. Using the notation introduced in Example 3, let $\gamma_{k}$ be specially chosen so that $\lambda_{N}\left(\Pi\left(H_{1}\left(\gamma_{k}\right)\right)\right) \leq \theta_{k} \lambda_{N}\left(\bigcup_{\Omega \in \mathcal{O}_{0}} \Omega\right)$, for each $k \in N$. By construction we have $\lambda_{N}\left(\Pi\left(H_{1}\left(\gamma_{k}\right)\right)\right)=\lambda_{N}\left(\Pi\left(H_{2}\left(\gamma_{k}\right)\right)\right)$ for all $k$, and that $\left\{\Pi\left(H_{i}\left(\gamma_{k}\right)\right) \mid k \in N, i=1,2\right\}$ is a family of mutually disjoint $N$-dimensional spherical discs contained in $\bigcup_{\Omega \in \mathcal{O}_{0}} \Omega$. Therefore,

$$
\begin{aligned}
\lambda_{N}\left(\bigcup_{\Omega \in \mathcal{O}_{0}} \Omega \backslash L_{\mathrm{J}}(G)\right) & \leq \sum_{k \in N} \sum_{i=1,2} \lambda_{N}\left(\Pi\left(H_{i}\left(\gamma_{k}\right)\right)\right) \\
& \leq 2 \sum_{k \in N} \theta_{k} \lambda_{N}\left(\bigcup_{\Omega \in \mathcal{O}_{0}} \Omega\right)<\lambda_{N}\left(\bigcup_{\Omega \in \mathcal{O}_{0}} \Omega\right),
\end{aligned}
$$

which shows that $L_{\mathrm{J}}(G)$ is of positive $N$-dimensional spherical Lebesgue measure.

\section{REFERENCES}

[1] T. Akaza and H. Furusawa, The exponent of convergence of Poincaré series of some Kleinian groups, Tôhoku Math. J. 32 (1980), 447-452.

[2 ] A. F. BEARDON, The Hausdorff dimension of singular sets of properly discontinuous groups, Amer. J. Math. 88 (1966), 722-736

[ 3 ] A. F. BeARDon, Inequalities for certain Fuchsian groups, Acta Math. 127 (1971), 221-258.

[4] A. F. Beardon, The Geometry of Discrete Groups, Springer Verlag, New York, 1983.

[ 5 ] C. J. Bishop, The linear escape limit set, Proc. Amer. Math. Soc. 132 (2004), 1385-1388.

[ 6 ] C. J. Bishop AND P. W. Jones, Hausdorff dimension and Kleinian groups, Acta Math. 56 (1997), 1-39.

[ 7 ] R. BRooks, The fundamental group and the spectrum of the Laplacian, Comment. Math. Helv. 56 (1981), 581-598.

[ 8 ] R. BRooks, The bottom of the spectrum of a Riemannian covering, J. Reine Angew. Math. 357 (1985), $101-114$.

[ 9 ] K. J. FAlconer, Fractal Geometry, Mathematical Foundations and Applications, J. Wiley, 1990.

[10] E. Hopf, Statistik der geodätischen Linien in Mannigfaltigkeiten negativer Krümmung, Ber. Verh. Sächs. Akad. Wiss. Leipzig 91 (1939), 261-304.

[11] T. LUNDH, Geodesics on quotient manifolds and their corresponding limit points, Michigan Math. J. 51 (2003), 279-304.

[12] K. MatsuZaKi, The Hausdorff dimension of the limit sets of infinitely generated Kleinian groups, Math. Proc. Cambridge Philos. Soc. 128 (2000), 123-139.

[13] K. MatsuZaki, Conservative action of Kleinian groups with respect to the Patterson-Sullivan measure, Comput. Methods Funct. Theory 2 (2002), 469-479. 
[14] K. MatsuZaki And M. TAniguchi, Hyperbolic manifolds and Kleinian groups, Oxford Math. Monographs, 1998.

[15] P. Mattila, Geometry of Sets and Measures in Euclidean Spaces. Fractals and Rectifiability, Cambridge University Press, Cambridge, 1995

[16] P. J. Nicholls, The Ergodic Theory of Discrete Groups, Cambridge University Press, Cambridge, 1989.

[17] J. R. PARKer And B. O. Stratmann, Kleinian groups with singly cusped parabolic fixed points, Kodai Math. J. 24 (2001), 169-206.

[18] S. J. Patterson, The exponent of convergence of Poincaré series, Monatsh. Math. 82 (1976), $297-315$.

[19] S. J. Patterson, Some examples of Fuchsian groups, Proc. London Math. Soc. (3) 39 (1979), $276-298$.

[20] S. J. PAtterson, Further remarks on the exponent of convergence of Poincaré series, Tôhoku Math. J. 35 (1983), 357-373.

[21] S. J. PAtTERSon, Lectures on measures on limit sets of Kleinian groups, Analytical and geometric aspects of hyperbolic space, editor D.B.A. Epstein, Cambridge University Press, Cambridge, 1987.

[22] Ch. Pommerenke, On the Green's function of Fuchsian groups, Ann. Acad. Sci. Fenn. A1 Math 2 (1976), 409-427.

[23] M. REES, Checking ergodicity of some geodesic flows with infinite Gibbs measure, Ergodic Theory Dynamical Systems 1 (1981), 107-133.

[24] M. REES, Divergence type of some subgroups of finitely generated Fuchsian groups, Ergodic Theory Dynamical Systems 1 (1981), 209-221.

[25] B. O. Stratmann, A note on counting cuspidal excursions, Annal. Acad. Sci. Fenn. 20 (1995), 359-372.

[26] B. O. Stratmann, The exponent of convergence of Kleinian groups; on a theorem of Bishop and Jones, preprint in Mathematica Gottingensis 1 (2001), 1-16; to appear in Proceedings of 'Fractal Geometry and Stochastics' (Friedrichroda, 2003).

[27] B. O. Stratmann, Fractal Geometry on Hyperbolic Manifolds, Mathematica Gottingensis 09 (2003), 1-20; to be published in Proceedings of the 'Janos Bolyai Conference on Hyperbolic Geometry' (Budapest, 2002); Kluwer Acad. Publ.

[28] D. Sullivan, The density at infinity of a discrete group of hyperbolic motions, Inst. Hautes Études Sci. Publ. Math. 50 (1979), 171-202.

[29] D. Sullivan, A finiteness theorem for cusps, Acta Math. 147 (1981), 289-299.

[30] D. Sullivan, On the ergodic theory at infinity of an arbitrary discrete group of hyperbolic motions, Riemann surfaces and related topics, Ann. of Math. Stud. 97 (1981), 465-496.

[31] P. TukiA, Conservative action and the horospheric limit set, Ann. Acad. Sci. Fenn. 22 (1997), 387-394.

[32] N. T. VAropoulos, Finitely generated Fuchsian groups, J. Reine Angew. Math. 375/376 (1987), 394-405.

[33] S. WAgOn, The Banach-Tarski paradox, Cambridge University Press, 1986.

[34] R. J. Zimmer, Ergodic theory and semisimple groups, Monographs in Mathematics, 81. Birkhäuser Verlag, Basel, 1984.

Mathematical Institute

UNIVERSITY OF BERN

SIDLERSTRASSE 5

CH-3012 BERN

SWITZERLAND

E-mail address: kurt.falk@math-stat.unibe.ch
Mathematical Institute

UNIVERSITY OF ST ANDREWS

NORTH HAUGH

ST ANDREWS KY16 9SS

UNITED KINGDOM

E-mail address: bos@st-and.ac.uk 